\title{
Phenylephrine-induced recruitable preload from the venous side
}

\author{
Rita Jacobs ${ }^{1} \cdot$ Stijn Lochy ${ }^{1,2} \cdot$ Manu L. N. G. Malbrain ${ }^{1,3}$
}

Received: 12 November 2018 / Accepted: 14 November 2018 / Published online: 26 November 2018 (c) The Author(s) 2018

\section{Shock is not always hypovolemia}

During the perioperative period, patients often suffer from hemodynamic instability, especially after major abdominal surgery, due to several pathophysiological processes: anesthetics, hypovolemia, surgical procedure, mechanical ventilation, cardiac co-morbidities and patient positioning [1]. Over the last decades we have used aggressive fluid resuscitation as a cornerstone in the treatment of shock, however, with only little evidence to support this [2-4]. Much of our current traditions of fluid resuscitation comes from experience in treating the blue stage of spasmodic cholera, where giving large amounts of aqueous and saline injections saved lives [5]. Ever since, it is deeply incorporated in our culture that shock must always equal hypovolemia. Fluid administration to maintain or restore circulation hence became an integrated part in the care for patients undergoing surgery, but also in critically ill patients admitted to the ICU.

However, avoiding hypervolemia is mandatory (especially in situations of capillary leak and global increased permeability syndrome), since excessive fluid administration usually leads to edema, increased inflammation and permeability and compromised tissue healing [6-9]. To avoid excessive fluid administration leading to edema and fluid overload, while maintaining an adequate mean arterial pressure (MAP), vasopressors may be required. It has to be noted that the term fluid overload has recently been questioned and hyperhydration or intravascular versus extravascular hypervolemia may be more adequate terms [9].

Manu L. N. G. Malbrain

manu.malbrain@uzbrussel.be

1 Intensive Care Department, University Hospital Brussels (UZB), Laarbeeklaan 101, 1090 Jette, Belgium

2 Cardiology Department, University Hospital Brussels (UZB), Jette, Belgium

3 Faculty of Medicine and Pharmacy, Vrije Universiteit Brussel (VUB), Brussels, Belgium

\section{Venoconstriction as recruitable preload source}

More than $70 \%$ of total blood volume (TBV) is located in the large veins, and increased vascular compliance (vasoplegia) like in sepsis or induced by anesthesia, can cause a substantial increase in TBV (with up to 80\%) [10]. This increased venous compliance and increased TBV in early sepsis may represent a recruitable source of preload, as veins are very sensitive to low doses of vasopressor $[11,12]$. These drugs directly convert unstressed blood volume to stressed blood volume while maintaining nearly normal venous elastance [11] (Fig. 1). Phenylephrine is widely used to treat intraoperative hypotension (via arterial and venous constriction). As a direct $\alpha$-adrenergic receptor agonist, phenylephrine predominantly increases the systemic vascular resistance (SVR), systolic arterial pressure, MAP and left ventricular afterload, however its effects on cardiac output (CO) remain controversial [13]. The impact of phenylephrine on $\mathrm{CO}$ seems to be associated to preload dependency, where it generates most often an increase in $\mathrm{CO}$ through increased venous return. However, when the heart is preload independent, phenylephrine generally induces a decrease in $\mathrm{CO}$, due to the increase in afterload [14-16].

Within this perspective, the recent publication by Wodack et al. elegantly described the effects of continuous administration of phenylephrine on the main determinants of $\mathrm{CO}$ [17]. The application of phenylephrine resulted in a significant increase in the volumetric preload variable global end-diastolic volume (GEDV), but not on the pressure-based preload variables central venous pressure (CVP) and an analog of mean systemic filling pressure (Pmsf). This effect is contributed to the effect of recruiting venous return from the venous capacitance pool, which then becomes available for the heart to generate stroke volume [18, 19]. This effect on preload volume was only observed after the first application of phenylephrine. However, a further increase in $\mathrm{CO}$ and cardiac contractility was observed, attributed to the positive inotropic effect of phenylephrine at higher dosages. 


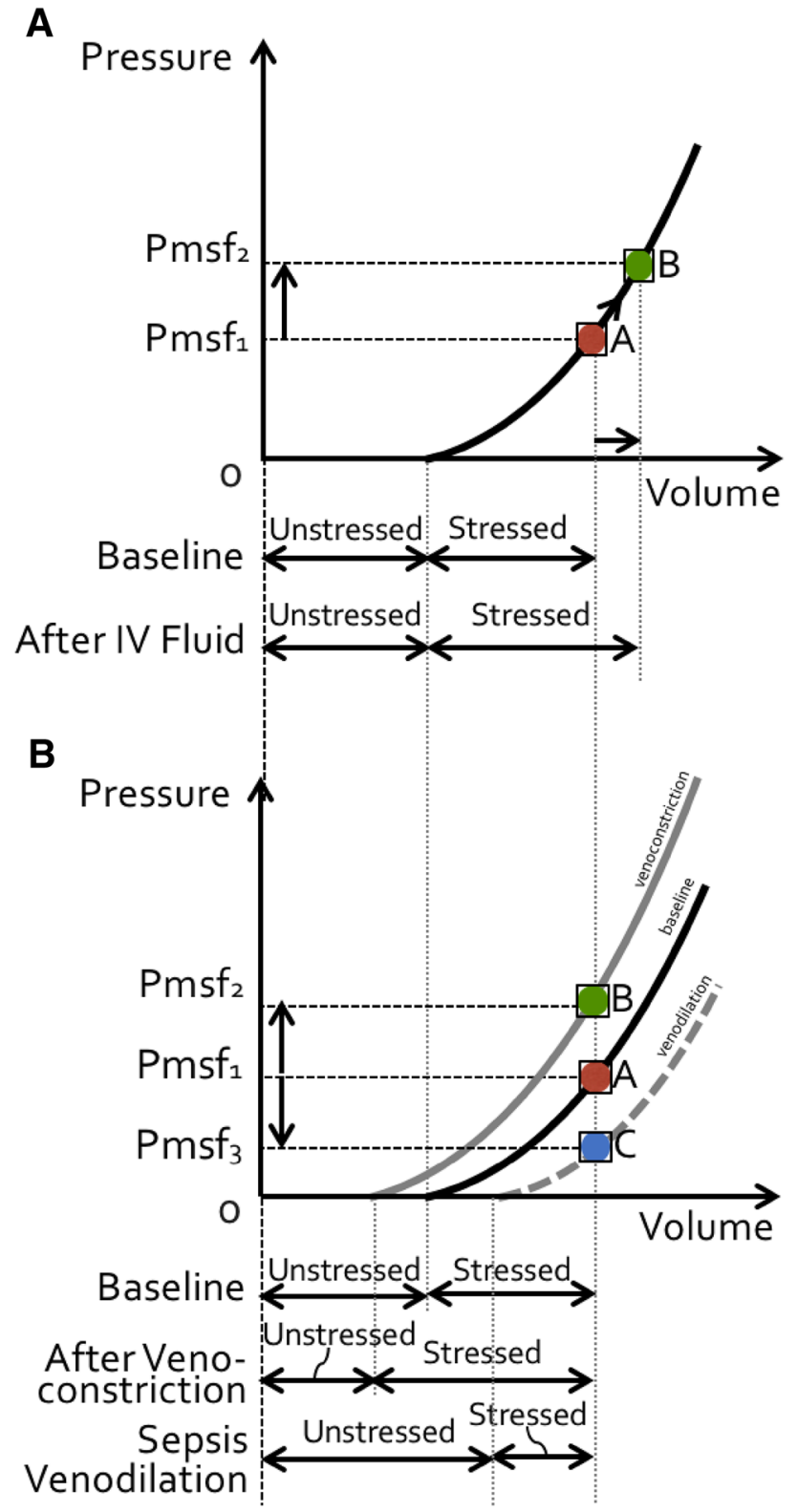

Fig. 1 Effect of fluid loading and venoconstriction on volume. a Effect of volume loading on mean systemic filling pressure (Pmsf) and (un)stressed volume. Administration of a fluid bolus increases $P$ msf (from $P$ msf1 to $P$ msf2, indicated respectively by position A (red dot) to B (green dot) on the pressure/volume curve). Unstressed volume remains constant while stressed volume increases. Total volume $=$ unstressed + stressed increases, carrying a risk for fluid overload. See text for explanation. b Effect of venoconstriction and venodilation on mean systemic filling pressure (Pmsf) and (un)stressed volume. Venoconstriction increases Pmsf (from Pmsf1 to Pmsf2, indicated respectively by position A (red dot) to B (green dot) on the pressure/volume curve). Unstressed volume decreases while stressed volume increases. Total volume $=$ unstressed + stressed remains constant, resulting in an auto-transfusion effect. Venodilation as seen in sepsis (vasoplegia) decreases Pmsf (from Pmsf1 to Pmsf3, indicated respectively by position A (red dot) to $\mathrm{C}$ (blue dot) on the pressure/ volume curve). Unstressed volume increases while stressed volume decreases. Total volume $=$ unstressed + stressed remains constant, resulting in an intravascular underfilling effect. See text for explanation

\section{All that glitters is not gold}

Despite the interesting results, some methodological weaknesses should be considered.

First, as only eight pigs were studied, results cannot be translated into clinical practice.

Second, stroke volume variation (SVV) measured by arterial pulse contour analysis below $10 \%$ was used to determine a state of relative fluid unresponsiveness. However, although far better than static variables of LV preload, studies comparing pulse pressure variation (PPV) with SVV, found PPV to have a better predictive ability than the SVV [20].

Third, a further increase in $\mathrm{CO}$, cardiac function index (CFI) and dPmax was attributed to a positive inotropic effect of phenylephrine. However, there was no correlation with echocardiographic measurements, the gold standard for assessment of LV contractility [21-23].

Fourth, another limitation is that phenylephrine changes vasomotor tone as well as venous capacitance. Vasopressors can significantly impair the ability of the dynamic preload indicators to predict fluid responsiveness, masking true intravascular volume deficit [24].

Fifth, the use of the mathematical modeling technique to determine Pmsf was based on MAP, CVP and CO. Any alteration in the measurement of these variables has an impact on the value of Pmsf [25]. Pmsf and CFI values were derived mathematically and are therefore, coupled with CO. Moreover, GEDV and CO values may also be mathematically coupled. Since CO increased with phenylephrine administration it is logic that GEDV increased following the formula:

$\mathrm{ITTV}=\mathrm{CO} \times M T T, \mathrm{PTV}=\mathrm{CO} \times D S T$ and

GEDV $=$ ITTV - PTV $=($ MTT - DST $) \times$ CO

with MTT mean transit time, DST down slope time, ITTV intrathoracic thermal volume, PTV pulmonary thermal volume. Thus, when CO increases because of this mathematical coupling GEDV will also increase [26].

Sixth, there was no radiographic confirmation of the tip of the central venous catheter, which may have affected the accuracy of the CVP measurements.

Seventh, it would have been interesting to see the gradual effects and the amount of fluid loading needed in order to obtain the state of relative fluid unresponsiveness. In the present study, animals were already hemodynamically stabilized prior to start of the protocol. 


\section{The case for mean systemic filling pressure}

Pmsf is the blood pressure throughout the vascular system at zero flow and offers information on vascular compliance, volume responsiveness and it allows the calculation of (un) stressed volume. For determination of mean circulatory filling pressure two other bedside methods are available, either based on inspiratory hold-derived venous return curves (Pmsf hold), or on arterial and venous pressure equilibration (Pmsf arm) [27].

Pmsf hold is based on the linear relation between CVP and venous return $\left(V_{\mathrm{R}}\right)$ :

$V_{\mathrm{R}}=(P m s f-C V P) / R V R$

where RVR is the resistance to $V_{\mathrm{R}}$. Hereby, the CVP is raised by performing a series of end-inspiratory hold maneuvers and $\mathrm{CO}$ is measured in the last three seconds of the $12 \mathrm{~s}$ inspiratory hold. After 7-10 s a steady state occurs when $V_{\mathrm{R}}=\mathrm{CO}$. By plotting the CVP and CO values, a $V_{\mathrm{R}}$ curve is constructed and the zero-flow pressure (Pmsf) extrapolated [28, 29].

As Pmsf is defined as the steady-state blood pressure during no-flow conditions, the arm is used to estimate $\boldsymbol{P m s f}$ arm. The upper arm is occluded to $50 \mathrm{mmHg}$ above systolic blood pressure, using a rapid cuff inflator or a pneumatic tourniquet. Measurements of arterial and venous pressures through a radial artery catheter and a peripheral venous cannula in the forearm are performed. When these two pressures equalize, Pmsf arm values are obtained [28, 29].

\section{Venous capacitance}

Most of the circulating TBV is located in the venous part, containing as stated previously approximately $70 \%$ of the body's blood volume. Venous vascular beds consist of unstressed (70\%) and stressed (30\%) volume. Stressed volume represents blood volume in excess of the unstressed volume, and its volume in relation to venous motor tone defines the Pmsf [10].

Capacitance is the total contained volume that can be contained at a given pressure, and consists of stressed and unstressed volume. Stressed volume can be increased by decreasing vascular capacitance, which means recruiting unstressed volume into stressed volume. This is the equivalent of an auto-transfusion. Removal of sympathetic drive (e.g. during vasoplegia in sepsis or during sympathicolysis) can withdraw this equivalent amount of stressed volume and lead to a marked fall in mean circulating filling pressure (Fig. 1).

Under normal conditions, the body's ability to rapidly vary unstressed volume is the primary means by which it alters venous return in response to changing metabolic demands [30].

The venous system contains $\alpha(1)$-adrenergic receptors, and trough stimulation by phenylephrine the splanchnic capacitance vessels constrict [13]. However, phenylephrine also increases the venous resistance draining this region and the net effect on venous return is determined by how much volume is recruited versus how much the downstream resistance increased [13, 30].

\section{Conclusions}

In conclusion, the large animal study performed by Wodack et al. on phenylephrine to treat perioperative hypotension is promising, since it does not only affect cardiac afterload, but also increases preload by shifting blood from the venous capacitance. This effect was confirmed by the observed increase in GEDV. Early application of vasopressors has the potential to reduce intravascular volume deficit by recruiting blood from the venous compartment (auto-transfusion effect), as well as to avoid the detrimental effects of fluid overload. Further studies are needed are to evaluate how these changes in management may affect outcomes.

\section{Compliance with ethical standards}

Conflict of interest MLNGM is Professor of Medicine at the Vrije Universiteit Brussel (VUB) and ICU Director at the University Hospital in Brussels (UZB), Belgium. He is founding President of WSACS (The Abdominal Compartment Society, http://www.wsacs.org) and current Treasurer, he is also member of the Medical Advisory Board of Getinge (formerly Pulsion Medical Systems) and Serenno Medical, and consults for ConvaTec, Acelity, Spiegelberg, and Holtech Medical. He is co-founder of the International Fluid Academy (IFA). The IFA is integrated within the not-for-profit charitable organization iMERiT, International Medical Education and Research Initiative, under Belgian law. The IFA website (http://www.fluidacademy.org) is now an official SMACC affiliated site (Social Media and Critical Care) and its content is based on the philosophy of FOAM (Free Open Access Medical education-\#FOAMed). The site recently received the HONcode quality label for medical education (https://www.healthonnet.org/HONco de/Conduct.html?HONConduct519739). The other authors have no potential conflicts of interest in relation to the contents of this paper.

Open Access This article is distributed under the terms of the Creative Commons Attribution 4.0 International License (http://creativeco mmons.org/licenses/by/4.0/), which permits unrestricted use, distribution, and reproduction in any medium, provided you give appropriate credit to the original author(s) and the source, provide a link to the Creative Commons license, and indicate if changes were made. 


\section{References}

1. Lonjaret L, Lairez O, Minville V, Geeraerts T. Optimal perioperative management of arterial blood pressure. Integr Blood Press Control. 2014;7:49-59.

2. Rhodes A, et al. Surviving sepsis campaign: international guidelines for management of sepsis and septic shock 2016. Intensive Care Med. 2017;43(3):304-77.

3. Barrier KM. Summary of the 2016 international surviving sepsis campaign: a clinician's guide. Crit Care Nurs Clin North Am. 2018;30(3):311-21.

4. Levy MM, Evans LE, Rhodes A. The surviving sepsis campaign bundle: 2018 update. Crit Care Med. 2018;46(6):997-1000.

5. Latta T. Letter from Dr Latta to the Secretary of the Central Board of Health, London, affording a view of the rationale and results of his practice in the treatment of cholera by aqueous and saline injections 1831-32. Lancet. 2013;42:274-277.

6. Noel-Morgan J, Muir WW. Anesthesia-associated relative hypovolemia: mechanisms, monitoring, and treatment considerations. Front Vet Sci. 2018;5:53.

7. Malbrain ML, Marik PE, Witters I, Cordemans C, Kirkpatrick AW, Roberts DJ, Van Regenmortel N. Fluid overload, de-resuscitation, and outcomes in critically ill or injured patients: a systematic review with suggestions for clinical practice. Anaesthesiol Intensive Ther. 2014;46(5):361-80.

8. Malbrain MLNG, Van Regenmortel N, Saugel B, De Tavernier B, Van Gaal PJ, Joannes-Boyau O, Teboul JL, Rice TW, Mythen M, Monnet X. Principles of fluid management and stewardship in septic shock: it is time to consider the four D's and the four phases of fluid therapy. Ann Intensive Care. 2018;8(1):66.

9. Vincent JL, Pinsky MR. We should avoid the term "fluid overload". Crit Care. 2012;22(1):214.

10. Drees JA, Rothe CF. Reflex venoconstriction and capacity vessel pressure-volume relationships in dogs. Circ Res. 1974;34(3):360-73.

11. Guyton AC, Lindsey AW, Bernathy B, Richardson T. Venous return at various right atrial pressures and the normal venous return curve. Am J Physiol. 1957;16:609-15.

12. Shen T, Baker K. Venous return and clinical hemodynamics: how the body works during acute hemorrhage. Adv Physiol Educ. 2015;39(4):267-71.

13. Thiele RH, Nemergut EC, Lynch C. The physiologic implications of isolated alpha(1) adrenergic stimulation. Anesth Analg. 2011;113(2):284-96.

14. Cannesson M, Jian Z, Chen G, Vu TQ, Hatib F. Effects of phenylephrine on cardiac output and venous return depend on the position of the heart on the Frank-Starling relationship. J Appl Physiol. 2012;113(2):281-9.

15. Rebet O, Andremont O, Gérard JL, Fellahi JL, Hanouz JL, Fischer MO. Preload dependency determines the effects of phenylephrine on cardiac output in anaesthetised patients: a prospective observational study. Eur J Anaesthesiol. 2016;33(9):638-44.

16. Kalmar AF, Allaert S, Pletinckx P, Maes JW, Heerman J, Vos JJ, Struys MMRF, Scheeren TWL. Phenylephrine increases cardiac output by raising cardiac preload in patients with anesthesia induced hypotension. J Clin Monit Comput. 2018. https://doi. org/10.1007/s10877-018-0126-3.
17. Wodack KH, Graessler MF, Nishimoto SA, Behem CR, Pinnschmidt HO, Punke MA, Monge-García MI, Trepte CJC, Reuter DA. Assessment of central hemodynamic effects of phenylephrine: an animal experiment. J Clin Monit Comput. 2018. https://doi.org/10.1007/s10877-018-0204-6.

18. Monnet X, Jabot J, Maizel J, Richard C, Teboul JL. Norepinephrine increases cardiac preload and reduces preload dependency assessed by passive leg raising in septic shock patients. Crit Care Med. 2011;39(4):689-94.

19. Giraud R, Siegenthaler N, Arroyo D, Bendjelid K. Impact of epinephrine and norepinephrine on two dynamic indices in a porcine hemorrhagic shock model. J Trauma Acute Care Surg. 2014;77(4):564-9 (quiz 650-1).

20. Preisman S, Kogan S, Berkenstadt H, Perel A. Predicting fluid responsiveness in patients undergoing cardiac surgery: functional haemodynamic parameters including the respiratory systolic variation test and static preload indicators. $\mathrm{Br} \mathrm{J}$ Anaesth. 2005;95(6):746-55.

21. Peeters Y, Bernards J, Mekeirele M, Hoffmann B, De Raes M, Malbrain ML. Hemodynamic monitoring: To calibrate or not to calibrate? Part 1-calibrated techniques. Anaesthesiol Intensive Ther. 2015;47(5):487-500.

22. Bernards J, Mekeirele M, Hoffmann B, Peeters Y, De Raes M, Malbrain ML. Hemodynamic monitoring: to calibrate or not to calibrate? Part 2-non-calibrated techniques. Anaesthesiol Intensive Ther. 2015;47(5):501-16.

23. Jalil BA, Cavallazzi R. Predicting fluid responsiveness: a review of literature and a guide for the clinician. Am J Emerg Med. 2018. https://doi.org/10.1016/j.ajem.2018.08.037.

24. Bouchacourt JP, Riva JA, Grignola JC. The increase of vasomotor tone avoids the ability of the dynamic preload indicators to estimate fluid responsiveness. BMC Anesthesiol. 2013;13(1):41.

25. Cecconi M, Aya HD, Geisen M, Ebm C, Fletcher N, Grounds RM, Rhodes A. Changes in the mean systemic filling pressure during a fluid challenge in postsurgical intensive care patients. Intensive Care Med. 2013;39(7):1299-305.

26. Hofkens PJ, Verrijcken A, Merveille K, Neirynck S, Van Regenmortel N, De laet I, Schoonheydt K, Dits H, Bein B, Huber W, Malbrain LMLNG. Common pitfalls and tips and tricks to get the most out of your transpulmonary thermodilution device: results of a survey and state-of-the-art review. Anaesthesiol Intensive Ther. 2015;47(2):89-116.

27. Wijnberge M, Sindhunata DP, Pinsky MR, Vlaar AP, Ouweneel E, Jansen JR, Veelo DP, Geerts BF. Estimating mean circulatory filling pressure in clinical practice: a systematic review comparing three bedside methods in the critically ill. Ann Intensive Care. 2018;8(1):73.

28. Maas JJ, Pinsky MR, Geerts BF, de Wilde RB, Jansen JR. Estimation of mean systemic filling pressure in postoperative cardiac surgery patients with three methods. Intensive Care Med. 2012;38(9):1452-60.

29. Arshed S, Pinsky MR. Applied physiology of fluid resuscitation in critical illness. Crit Care Clin. 2018;2:267-277.

30. Magder S. Volume and its relationship to cardiac output and venous return. Crit Care. 2016;20:271. 